and Tour de Cure to screen 240 patients, with plans to roll out nationally.

\section{T-NHL SUBTYPES AND CLINICAL PERSPECTIVE}

\author{
H. Miles Prince ${ }^{1,2}$ \\ ${ }^{1}$ Peter MacCallum Cancer Centre, Melbourne, Vic, Australia; \\ and ${ }^{2}$ Epworth Healthcare, Vic, Australia
}

Peripheral T-cell lymphomas (PTCL) are a distinct pathological entity with clinical advancements lagging behind its B-cell lymphoma counterpart. A significant proportion (15-20\%) of PTCL cases are misdiagnosed as reactive or a different lymphoma subtype. An accurate diagnosis routinely requires analysis of the immunophenotype in conjunction with cellular morphology, analysis of lymph node architecture and molecular genetic analysis. Recent major advances in genomic studies examining gene expression profiling (GEP) and the genetic landscape of T-cell and NK-cell neoplasms have resulted in diagnostic, prognostic and therapeutic implications and has consequently led to revisions in the classification of PTCLs and introduction of new provisional entities. Incorporating these molecular and genetic markers, the latest 2016 WHO classification, an update of the current fourth edition, has identified 25 definitive and 6 provisional entities and further groups these lymphomas according to their usual presentation into disseminated disease (leukaemias), predominantly nodal, predominantly extra-nodal or cutaneous lymphomas. Nodal PTCLs represent the most prevalent PTCL subtype and include peripheral T-cell lymphoma - not otherwise specified (PTCL-NOS), angioimmunoblastic T-cell lymphoma (AITL), follicular T-cell lymphoma (FTCL), nodal PTCL with T follicular helper (TFH) phenotype, and systemic anaplastic large cell lymphoma (sALCL) either ALK-positive or ALK-negative.

Due to better understanding of the cell of origin in T-cell lymphomas, AITL, FTCL and PTCL with TFH phenotype have been recognised as being derived from TFH cells as these neoplastic cells express at least two or three TFH cell antigens which include CD279/PD1, CD10, BCL6, CXCL13, ICOS, SAP and CCR5. In addition to the common cell of origin, these lymphomas share pathological and clinical features as well as recurrent genetic abnormalities such as mutations in TET2, IDH2, DNMT3A, RHOA and CD28, and gene fusions such as $I T K-S Y K$ and CTLA-CD28. Such features suggest that these lymphomas belong to the same spectrum of disease and are therefore now unified under a common heading.

PTCL-NOS encompasses those which do not match the classification of other PTCL categories. This subtype mostly has a CD4+/CD8 - phenotype and shows extreme cytological and phenotypical heterogeneity but GEP have identified at least three subgroups characterised by overexpression of GATA3, TBX21 and cytotoxic genes associated with varied clinical behaviour. Systemic ALCLs have a primarily aggressive nodal presentation and are further subdivided to ALK-positive or ALK-negative based on whether they carry the nucleoplasmin (NPM) anaplastic lymphoma kinase (ALK) translocation between chromosome 2 and 5, designated as $\mathrm{t}(2 ; 5)$. We now know that a subset of ALCL, ALK-negative cases harbour rearrangement at the locus containing DUSP22 and IRF4 in chromosome 6p25, which induces down regulation of DUSP22, a dual specific phosphatase that inhibits TCR signalling. Another subset of ALK-negative
ALCL has rearrangements in TP63. Clear distinction of PTCL with high CD30 expression from ALCL, ALK-negative have also been possible through GEP and as such, the former is no longer considered a provisional entity.

Breast implant associated ALCL, a unique form of ALCL, ALKnegative arising in association with breast implants, is increasingly being recognised and has been included as a provisional entity. Cutaneous ALCL (cALCL), ALK-negative, the fourth subtype, is an indolent form limited to the skin.

Whilst notorious to have an aggressive clinical course and an extremely poor overall prognosis, clinicians are constantly challenged with low rates of complete remissions, early relapses and failures to achieve long-term remissions despite aggressive first-line chemotherapy, in the majority of patients. More recently, improved understanding of disease biology and the molecular profile of specific PTCL subtypes has opened the door to the potential of more targeted therapies with a number of new biological therapies approved by regulatory agencies in the last few years.

\section{INTERACTIVE TRANSFUSION CASES: A DAY IN THE LIFE OF A TRANSFUSION MEDICINE SPECIALIST}

\author{
Anastazia Keegan ${ }^{1,2}$ \\ ${ }^{1}$ Department of Haematology, Nepean Hospital, Kingswood, \\ NSW, Australia; and ${ }^{2}$ Transfusion Policy and Education, \\ Australian Red Cross Lifeblood, Australia
}

Join Dr Keegan as she guides you through a range of challenging transfusion scenarios focusing on the laboratory investigations required to manage these complex clinical situations.

This interactive session will explore the complexity of the RhD blood group, highlight a rare but life-threatening complication of transfusion, provide an approach to the 'untransfusable' patient and empower you to make hard decisions confidently during an emergency.

\section{PATIENT BLOOD MANAGEMENT - A REVIEW OF ACCEPTED PRACTICE; WITH A FOCUS ON OPTIMISING PATIENT OUTCOMES DURING THE CURRENT COVID-19 PANDEMIC}

\author{
Bryony Ross $^{1}$, Lisa Clarke ${ }^{2}$, Peter Freeman ${ }^{3}$ \\ ${ }^{1}$ Department of Haematology, The Children's Hospital at \\ Westmead, Sydney, NSW, Australia; ${ }^{2}$ Transfusion Medicine \\ Specialist, Australian Red Cross Lifeblood, Sydney, NSW, \\ Australia; and ${ }^{3}$ Patient Blood Management Officer, Hunter New \\ England Health, Newcastle, NSW, Australia
}

Patient blood management (PBM) has long been accepted as a multidisciplinary, patient-centred, evidence based approach to conservatively manage a patient's own blood with the goal of maintaining haemoglobin concentration, optimising haemostasis and minimising blood loss in an effort to improve patient outcome. $^{1}$

This rationale accepts that the patient's own blood is a valuable resource to be conserved and managed whilst acknowledging that altruistically donated blood is a unique and costly resource. It holds that blood transfusions are a therapy to be used only when there is evidence for likely benefit; and that this should be the standard of care for all patients. 
The overarching focus of PBM has been driven by a number of factors including: ${ }^{2}$

- The risks associated with blood transfusion.

- Rising costs, both direct and indirect, associated with provision and transfusion of allogeneic blood.

- Ongoing challenges of maintaining an adequate blood supply. These factors will be evaluated, with an overview of the current literature, a particular focus on partnering with patients, and discussion surrounding the unique challenges of implementing PBM practices to improve patient outcomes during a crisis situation, including the current COVID-19 pandemic. $^{3}$

\section{References}

1. Mueller MM, Van Remoortel H, Meybohm P, et al. Patient blood management: recommendations from the 2018 Frankfurt Consensus Conference. JAMA 2019; 321: 983-97.

2. Thomson A, Farmer S, Hofmann A, et al. Patient blood management - a new paradigm for transfusion medicine? ISBT Sci Ser 2009; 4: 423-35.

3. Baron DM, Franchini M, Goobie SM, et al. Patient blood management during the COVID-19 pandemic: a narrative review. Anaesthesia 2020; 75: 1105-13. 